International Electronic Journal of Algebra

Volume 21 (2017) 103-120

\title{
FINITE LATTICES OF PRERADICALS AND FINITE REPRESENTATION TYPE RINGS
}

\author{
Rogelio Fernández-Alonso and Dolors Herbera \\ Received: 8 April 2016; Revised: 12 September 2016 \\ Communicated by Abdullah Harmancı
}

\begin{abstract}
In this paper we study some classes of rings which have a finite lattice of preradicals. We characterize commutative rings with this condition as finite representation type rings, i.e., artinian principal ideal rings. In general, it is easy to see that the lattice of preradicals of a left pure semisimple ring is a set, but it may be infinite. In fact, for a finite dimensional path algebra $\Lambda$ over an algebraically closed field we prove that $\Lambda$-pr is finite if and only if its quiver is a disjoint union of finite quivers of type $\mathbb{A}_{n}$; hence there are path algebras of finite representation type such that its lattice of preradicals is an infinite set. As an example, we describe the lattice of preradicals over $\Lambda=k Q$ when $Q$ is of type $\mathbb{A}_{n}$ and it has the canonical orientation.
\end{abstract}

Mathematics Subject Classification (2010): 16G60, 16S90

Keywords: Preradical, finite representation type, finite lattice

\section{Introduction}

A good tool to make a description for the lattice of preradicals over the category of left modules over a ring $R$ is to have a set $\mathcal{I}$ of left modules such that every module is isomorphic to a direct sum of modules in $\mathcal{I}$. By results due to Gruson and Jensen (see [10]), in this case, the set $\mathcal{I}$ can be taken to consist of finitely presented and indecomposable modules and, therefore, $R$ is a left pure semisimple ring. Such is the case, for example, of the following classes of rings:

(1) Köthe rings,

(2) semisimple artinian rings (see [12]),

(3) local uniserial rings (see [7]),

(4) artin algebras of finite representation type (see [3]) or, in general,

(5) rings of finite representation type.

We recall that it is an open question, known as the pure semisimple conjecture, whether a left pure semisimple ring has finite representation type.

In Section 3 we prove that for a commutative ring $R$, the lattice of preradicals is finite if and only if $R$ has finite representation type, that is, if and only if $R$ is 
an artinian principal ideal ring. Since the lattice of preradicals of such a ring is already described in [7], a consequence is that all finite lattices of preradicals for commutative rings are known.

In the non-commutative case, there are finite representation type rings with an infinite lattice of preradicals. We prove in Section 4 that among all finitedimensional hereditary artin algebras over an algebraically closed field, only those that are Morita equivalent to a finite product of path algebras over quivers of type $\mathbb{A}_{n}$ have a finite lattice of preradicals. Finally, in Section 5 we describe some properties of this finite lattice when the quiver of type $\mathbb{A}_{n}$ has the canonical orientation. Our tool-kit to prove these results is the Auslander-Reiten theory applied to the study of the lattice of preradicals of path algebras.

\section{Lattices and preradicals}

In this section we list some definitions and propositions that are used later, together with the references where they can be found.

For basic definitions and results on posets and lattices see, for example, [5] and [9]. We just recall a theorem that will be used later on this paper.

The following theorem gives equivalent conditions for the Jordan-Dedekind property in finite posets (see [5, p. 11] and [9, p. 233]). It will be useful in Section 5 to describe a particular lattice of preradicals.

Theorem 2.1. For a finite poset $P$ with least element $\widehat{0}$ and greatest element $\widehat{1}$ the following conditions are equivalent:

(a) For any $x, y \in P$ all maximal chains between $x$ and $y$ have the same length.

(b) All maximal chains in $P$ have the same length.

(c) There exists a function $\rho: P \longrightarrow \mathbb{N}$ such that:

(i) $\rho(\widehat{0})=0$.

(ii) For any $x, y \in P$ if $y$ covers $x$ then $\rho(y)=\rho(x)+1$.

As in [14, p. 99], a finite poset $P$ which satisfies any of the conditions of Theorem 2.1 is called graded and it has rank $n$ if $\rho(\widehat{1})=n$.

Let $R$ be an associative ring with identity. $R$-Mod denotes the category of all unital left $R$-modules. The unadorned term $R$-module means left $R$-module.

For $N, M \in R$-Mod we write $N \leq_{f i} M$ meaning $N$ is a fully invariant submodule of $M$. For each $M \in R$-Mod we denote by $\mathcal{L}(M)$ the lattice of submodules of $M$ and by $\mathcal{L}_{f i}(M)$ the lattice of fully invariant submodules of $M$. 
The terminology and basic concepts about preradicals can be found in [12] and [13]. A (left) preradical over the $\operatorname{ring} R$ is a subfunctor of the identity functor on $R$-Mod. We denote by $R$-pr the class of all preradicals over $R$. Similarly, we define right preradicals and denote by pr- $R$ the class which consists of them. There is a natural partial ordering in $R$-pr given by $\sigma \preceq \tau$ if $\sigma(M) \leq \tau(M)$ for every $M \in R$-Mod.

The following result is immediate from the definition of preradical, and very important to describe lattices of preradicals.

Proposition 2.2. Let $\sigma \in R$-pr and let $\left\{M_{\gamma}\right\}_{\gamma \in I}$ be a family of $R$-modules. Then $\sigma\left(\bigoplus_{\gamma \in I} M_{\gamma}\right)=\bigoplus_{\gamma \in I} \sigma\left(M_{\gamma}\right)$.

There are four usual operations in $R$-pr, namely $\wedge, \vee, \cdot$ and : which are defined as follows, for $\sigma, \tau \in R$-pr and $M \in R$-Mod.

(1) $(\sigma \wedge \tau)(M)=\sigma(M) \cap \tau(M)$,

(2) $(\sigma \vee \tau)(M)=\sigma(M)+\tau(M)$,

(3) $(\sigma \tau)(M)=\sigma(\tau(M))$ and

(4) $(\sigma: \tau)(M)$ is such that $(\sigma: \tau)(M) / \sigma(M)=\tau(M / \sigma(M))$.

The meet $\wedge$ and join $\vee$ can be defined for arbitrary families of preradicals as in [12]. This makes sense because for any left $R$-module $M$, a sum (or an intersection) of submodules of $M$ which is indexed by a class can also be indexed by a set. With the partial ordering described above, $R$-pr results a complete big lattice, i.e. a class (not necessarily a set) having joins and meets for arbitrary families (indexed by a class) of elements. The smallest and largest elements of $R$-pr, which are the zero functor and the identity functor of $R$-Mod, are denoted respectively by $\widehat{0}$ and $\hat{1}$. This big lattice is atomic and co-atomic; the atoms and co-atoms are described below. The operation "." is called product and the operation ":" is called coproduct.

Some preradicals are of particular interest, such as idempotent preradicals, radicals, left exact preradicals and t-radicals. For basic definitions and results on these classes of preradicals see [15, VI.\$1], and [4] or [12].

The following types of preradicals have special importance.

Definition 2.3. [12, Definition 4] Let $N$ be a fully invariant submodule of $M$. The preradicals $\alpha_{N}^{M}$ and $\omega_{N}^{M}$ are defined as follows. Let $K \in R$-Mod.

$$
\begin{gathered}
\alpha_{N}^{M}(K)=\sum\left\{f(N) \mid f \in \operatorname{Hom}_{R}(M, K)\right\} \\
\omega_{N}^{M}(K)=\bigcap\left\{f^{-1}(N) \mid f \in \operatorname{Hom}_{R}(K, M)\right\} .
\end{gathered}
$$

A preradical is called an alpha preradical (omega preradical) if it is of the form $\alpha_{N}^{M}$ $\left(\omega_{N}^{M}\right)$ for some $M \in R$-Mod and $N \leq_{f i} M$. 
Every preradical can be expressed in terms of alpha and omega preradicals.

Remark 2.4. [12, Proposition 5] Let $\sigma \in R$-pr and $M, N \in R$-Mod. Then $\sigma(M)=$ $N$ if and only if $N$ is a fully invariant submodule of $M$ and $\alpha_{N}^{M} \preceq \sigma \preceq \omega_{N}^{M}$.

The previous remark says, in particular, that for a fully invariant submodule $N$ of $M, \alpha_{N}^{M}$ is the least preradical (and $\omega_{N}^{M}$ is the greatest preradical) that assigns $N$ to $M$.

Remark 2.5. If $K \leq N \leq M$ with $K$ and $N$ fully invariant in $M$ then $\alpha_{K}^{M} \preceq \alpha_{N}^{M}$ and $\omega_{K}^{M} \preceq \omega_{N}^{M}$.

The atoms and coatoms of $R$-pr are, respectively, $\left\{\alpha_{S}^{E(S)} \mid S \in R\right.$-simp $\}$ and $\left\{\omega_{I}^{R} \mid I\right.$ is a maximal ideal of $\left.R\right\}$ (see Theorem 7 of [12]).

We can list some situations in which the lattice of preradicals can be described.

Examples 2.6. (1) [4, Proposition I.9.1] Let $R=\prod_{i=1}^{n} R_{i}$. Then $R$-pr is lattice isomorphic to the product of the lattices $R_{i}-p r$.

(2) [4, Proposition I.9.2] Let $R$ and $S$ be Morita equivalent rings. Then the lattices $R$-pr and $S$-pr are isomorphic. In particular, if $S=\mathbb{M}_{n}(R)$ is the full ring of $n \times n$ matrices over $R$ then the lattices $R$-pr and $S$-pr are isomorphic.

(3) [12, Theorem 11] $R$ is a semisimple artinian ring with $n$ non-isomorphic simple modules if and only if $R$-pr is a finite Boolean lattice with $2^{n}$ elements.

(4) [7, Theorem 5.1] For any local uniserial ring $R$ with composition length $n$, $R$-pr is a finite distributive lattice with $2^{n}$ elements. As a consequence, if $R$ is an artinian principal ideal ring then $R$-pr is a finite distributive lattice with $2^{m}$ elements, for some $m \geq 1$.

\section{Pure semisimple and finite representation type rings}

The following definitions can be found in [11].

Definition 3.1. A ring $R$ is said to have finite representation type if it is left artinian and if there are, up to isomorphism, finitely many indecomposable finitely generated left $R$-modules.

A ring $R$ is said to be left pure semisimple if every left $R$-module is pure-injective or, equivalently, if every left $R$-module is pure-projective.

The condition of having finite representation type is actually left-right symmetric, as was shown by Eisenbud and Griffith [6]. 
It is a long standing open question, known as the pure semisimple conjecture, whether a left pure-semisimple is also right pure semisimple. We refer to [11] for an excellent survey on this question.

For further quoting we state the following theorem, which summarizes the characterizations of pure semisimple rings that will be useful to us, as well as the relation between pure semisimple rings and rings of finite representation type.

Theorem 3.2. For a ring $R$ the following conditions are equivalent:

(a) $R$ is left pure semisimple.

(b) Every left $R$-module is a direct sum of left finitely generated $R$-modules.

(c) Every left $R$-module is a direct sum of left indecomposable (finitely generated) $R$-modules.

(d) Every indecomposable left $R$-module is finitely presented.

(e) There exists a set $\mathcal{I}$ of left $R$-modules such that any left $R$-module is isomorphic to a direct sum of modules in $\mathcal{I}$.

If, in addition, $R$ is right pure semisimple then the above statements are further equivalent to

(f) $R$ has finite representation type.

(g) All left modules have finite length over its endomorphism ring.

In the case of a commutative ring we can add other equivalent conditions to Theorem 3.2. Recall that an artinian principal ideal ring is a ring which is both left and right artinian and a left and right principal ideal ring.

Theorem 3.3. Let $R$ be a commutative ring. Then $R$ has finite representation type if and only if $R$ is an artinian principal ideal ring.

We recall the following basis-free definition of finite matrix subgroups.

Definition 3.4. Let $R$ be a ring. Let $(X, x)$ be a pointed $R$-module, i.e. a left $R$-module $X$ and a "point" $x \in X$. We let $H_{X, x}(M)$ be the image of the map $\varepsilon_{x}: \operatorname{Hom}_{R}(X, M) \rightarrow M$ given by $\varepsilon_{x}(h)=(x) h$. Clearly, $H_{X, x}$ can be seen as a subfunctor of the forgetful functor $R$-Mod $\rightarrow \mathbb{Z}$-Mod that commutes with products. We call $H_{X, x}$ a matrix subgroup. If the module $X$ is finitely presented then $H_{X, x}$ is said to be a finite matrix subgroup.

The characterizations in Theorem 3.2 depend on the characterization of $\Sigma$-pureinjective modules as the modules satisfying the descending chain condition on matrix subgroups. For commutative rings, a matrix subgroup is a preradical (that commutes with products) so this yields the following characterization of rings of finite representation type in terms of the lattice of preradicals. 
Theorem 3.5. For a commutative ring $R$ the following conditions are equivalent:

(a) $R$ is an artinian principal ideal ring.

(b) $R$-pr is finite.

(c) R-pr is an artinian (and/or noetherian) lattice.

(d) $R$ is a ring of finite representation type.

Proof. $(a) \Leftrightarrow(d)$ Follows from Theorem 3.3. It is clear that $(b)$ implies $(c)$.

$(c) \Rightarrow(d)$ If $R$-pr is artinian then, by Remark 2.4, every module $M$ has an artinian and noetherian lattice of fully invariant submodules. Since $R$ is commutative, for each $M \in R$-Mod, every $\operatorname{End}_{R}(M)$-submodule of $M$ is also a fully invariant $R$-submodule of $M$. Therefore every $R$-module is artinian over its endomorphism ring and, hence, pure injective. By Theorem 3.2, $R$ has finite representation type.

If $R$-pr is noetherian, then any module satisfies the ascending chain condition on finite matrix subgroups and by [16, Theorem 6$]$ also the descending chain condition on finite matrix subgroups. Therefore $(d)$ also holds.

$(a) \Rightarrow(b)$ As an artinian principal ideal ring is a finite product of local artinian principal ideal rings, the implication follows from Examples 2.6.

Example 3.6. Let $R=\mathbb{Z}_{2} \ltimes \mathbb{Z}_{2}^{2}$ be the trivial extension of $\mathbb{Z}_{2}$ by $\mathbb{Z}_{2}^{2}$. $R$ is a commutative finite local ring with 8 elements, but it is not a principal ideal ring, so $R$-pr is infinite and neither artinian, nor noetherian by Theorem 3.5.

Let $R$ be a ring, and assume that we can fix a set $\mathcal{I}$ of left $R$-modules such that any module is isomorphic to a direct sum of copies of modules in $\mathcal{I}$. By Theorem 3.2, if such a set $\mathcal{I}$ exists then $R$ is a left pure semisimple ring and $\mathcal{I}$ can be taken to be a set of representatives of isomorphism classes of finitely presented indecomposable left $R$-modules.

Let $N \in \prod_{X \in \mathcal{I}} \mathcal{L}(X)$. For simplicity, we write $N_{X}$ instead of $N(X)$ and $\left(N_{X}\right)_{X \in \mathcal{I}}$ instead of $N$. The following concept generalizes fully invariant submodules.

Definition 3.7. Let $N \in \prod_{X \in \mathcal{I}} \mathcal{L}(X)$. We say that $N$ is an $\underline{\mathcal{I} \text {-compatible set }}$ of submodules if for each $X, Y \in \mathcal{I}$ and $f\left(N_{X}\right) \leq N_{Y}$ for each homomorphism $f: X \rightarrow$ $Y$.

The family of all $\mathcal{I}$-compatible sets of submodules will be denoted as $\mathcal{C}_{\mathcal{I}}^{R}$. The set $\mathcal{C}_{\mathcal{I}}^{R}$ is a partially ordered set with the component-wise order. That is $\left(N_{X}\right)_{X \in \mathcal{I}} \leq$ $\left(N_{X}^{\prime}\right)_{X \in \mathcal{I}}$ if and only if for any $X \in \mathcal{I}, N_{X} \subseteq N_{X}^{\prime}$. It is easy to see that $\mathcal{C}_{\mathcal{I}}^{R}$ is in fact a lattice. 
Remark 3.8. Notice that:

(1) If $N \in \mathcal{C}_{\mathcal{I}}^{R}$ then $N_{X} \leq_{f i} X$ for each $X \in \mathcal{I}$.

(2) $\mathcal{C}_{\mathcal{I}}^{R}$ is a complete sublattice of $\prod_{X \in \mathcal{I}} \mathcal{L}(X)$.

Lemma 3.9. If $N \in \mathcal{C}_{\mathcal{I}}^{R}$ then $\left(\bigvee_{X \in \mathcal{I}} \alpha_{N_{X}}^{X}\right)(Y)=N_{Y}$ for each $Y \in \mathcal{I}$.

Proof. By Definition 2.3 and since $N \in \mathcal{C}_{\mathcal{I}}^{R}, \alpha_{N_{X}}^{X}(Y) \leq N_{Y}$ for each $X, Y \in \mathcal{I}$. Therefore $\left(\bigvee_{X \in \mathcal{I}} \alpha_{N_{X}}^{X}\right)(Y) \leq N_{Y}$. Conversely, by Remark 2.4, $N_{Y}=\alpha_{N_{Y}}^{Y}(Y) \leq$ $\left(\bigvee_{X \in \mathcal{I}} \alpha_{N_{X}}^{X}\right)(Y)$

As a consequence we have a functor between partially ordered sets. $\lambda_{R}: R$-pr $\rightarrow \mathcal{C}_{\mathcal{I}}^{R}$ such that for each $\sigma \in R$-pr, $\lambda_{R}(\sigma)$ is the $\mathcal{I}$-compatible set such that for each $X \in \mathcal{I}, \lambda_{R}(\sigma)_{X}=\sigma(X)$. Also let $\mu_{R}: \mathcal{C}_{\mathcal{I}}^{R} \rightarrow R$-pr be such that for each $N \in$ $\mathcal{C}_{\mathcal{I}}^{R}, \mu_{R}(N)=\bigvee_{X \in \mathcal{I}} \alpha_{N_{X}}^{X}$

The definition of order in $R$-pr implies that $\lambda_{R}$ is indeed a functor, and Remark 2.5 implies that $\mu_{R}$ is an order morphism. By Lemma $3.9, \lambda_{R} \mu_{R}=1_{\mathcal{C}_{I}^{R}}$. We show in the next result that one is inverse of each other.

Proposition 3.10. Let $R$ be a left pure-semisimple ring, and fix a set $\mathcal{I}$ of left $R$-modules such that any left $R$-module is isomorphic to a direct sum of copies of modules in $\mathcal{I}$. The functor $\lambda_{R}: R-p r \rightarrow \mathcal{C}_{\mathcal{I}}^{R}$ is an equivalence with inverse $\mu_{R}$. Therefore $R$-pr and $\mathcal{C}_{\mathcal{I}}^{R}$ are isomorphic lattices.

Proof. We have only to prove that $\mu_{R} \lambda_{R}=1_{R \text {-pr }}$. For each $M \in R$-Mod we can write $M=\bigoplus_{X \in \mathcal{I}} X^{\left(\kappa_{X, M}\right)}$, for some cardinals $\kappa_{X, M}$. Let $\sigma \in R$-pr. Since $\sigma=\bigvee\left\{\alpha_{\sigma(M)}^{M} \mid M \in R\right.$-Mod $\}$, Proposition 2.2 yields $\sigma=\bigvee_{X \in \mathcal{I}} \alpha_{\sigma(X)}^{X}=\mu_{R}\left(\lambda_{R}(\sigma)\right)$ We conclude that $\lambda_{R}$ and $\mu_{R}$ are order, and hence lattice isomorphisms, one inverse to each other.

As a consequence we have the following important property for left pure semisimple rings.

Corollary 3.11. Let $R$ be a left pure semisimple ring then the class $R$-pr is a set. If the set $\mathcal{I}$ of indecomposable finitely presented modules is finite, that is, if $R$ is of finite representation type, and $\mathcal{L}(X)$ is finite for each $X \in \mathcal{I}$ then $R$-pr is finite.

The class of rings such that $R$-pr is a set is not determined. Our guess is that it might well coincide with the class of left pure-semisimple rings. 
Corollary 3.12. Let $R$ be a ring of finite representation type. Let $U$ be a left $R$-module that is an injective cogenerator of $R$ with finite dual Goldie dimension. Set $T=\operatorname{End}_{R}(U)$, then $R$-pr is anti-isomorphic to pr-T.

Proof. By hypothesis, ${ }_{R} U$ is linearly compact. Therefore, the bimodule ${ }_{R} U_{T}$ induces a Morita duality between $R$ and $T$. Therefore $T$ is a right artinian ring. By a well known result of Auslander [2, Theorem 3.1], for a right artinian ring $T$ the condition of being of finite representation type can be characterized in terms of sequences of morphisms between finitely presented right $T$ modules. This condition is preserved via Morita duality, so $T$ is also a ring of finite representation type.

Let $\mathcal{I}$ be a set of representatives of the indecomposable finitely generated left $R$-modules. Since the duality takes indecomposable modules to indecomposable modules,

$$
\mathcal{J}=\operatorname{Hom}_{R}(\mathcal{I}, U)=\left\{\operatorname{Hom}_{R}(X, U) \mid X \in \mathcal{I}\right\}
$$

is a set of representatives of the indecomposable right $T$-modules.

The duality gives a lattice anti-isomorphism

$$
\varphi: \mathcal{C}_{\mathcal{I}}^{R} \rightarrow \mathcal{C}_{\mathcal{J}}^{T}
$$

defined by $\varphi\left(N_{X}\right)_{X \in \mathcal{I}}=\left(M_{\operatorname{Hom}_{R}(X, U)}\right)_{X \in \mathcal{I}}$ with

$$
M_{\mathrm{Hom}_{R}(X, U)}=\left\{\omega \in \operatorname{Hom}_{R}(X, U) \mid \omega\left(N_{X}\right)=0\right\}
$$

for each $X \in \mathcal{I}$. In view of Proposition 3.10, $\varphi$ yields the claimed lattice antiisomorphism between $R$-pr and pr- $T$.

\section{Finite lattices of preradicals for path algebras}

For basic definitions and results on artin algebras, quivers and their representations the reader can see [1] or [3]. Let us first recall some basic facts about path algebras that will be useful for this paper.

Remark 4.1. Let $Q=\left(Q_{0}, Q_{1}\right)$ be a finite quiver, with set $Q_{0}$ of $n$ vertices and a finite arrows set $Q_{1}$. We assume that $Q$ has not oriented cycles. Let $\Lambda=k Q$ be the path algebra of the quiver $Q$ over the field $k$. Then:

(1) If $e_{i}$ is the trivial path corresponding to the vertex $i$ then $\left\{\Lambda e_{1}, \ldots, \Lambda e_{n}\right\}$ is a complete irrendundant set of projective indecomposable $\Lambda$-modules.

(2) For each $i, j \in Q_{0}, e_{j} \Lambda e_{i}$ has as a $k$-basis all the paths from $i$ to $j$.

(3) In particular for each $i \in Q_{0}$ we have $\operatorname{End}_{\Lambda}\left(\Lambda e_{i}\right) \cong e_{i} \Lambda e_{i}=k e_{i} \cong k$. 
Now we recall the famous theorem of Gabriel [8] which states that there is a oneto-one correspondence between indecomposable basic finite-dimensional hereditary algebras over an algebraically closed field, and connected finite quivers without oriented cycles. See also [3, Corollary III.1.10].

Theorem 4.2. [8] Let $\Lambda$ be an indecomposable basic finite-dimensional algebra over an algebraically closed field $k$. Then there is a connected finite quiver $Q$ and a finitely generated ideal $I<k Q$ such that $\Lambda \cong k Q / I$. Moreover, if $\Lambda$ is hereditary then $Q$ has no oriented cycles and $I=0$. The quiver $Q$ is uniquely determined by $\Lambda$ and is called the Gabriel quiver of $\Lambda$.

The Gabriel quivers associated to an artin algebra of finite representation type are classified, according to the following theorem also due to Gabriel.

Theorem 4.3. [3, VIII, Theorem 5.5] Let $\Lambda$ be an indecomposable basic finitedimensional hereditary algebra over an algebraically closed field $k$, and let $Q$ be the Gabriel quiver of $\Lambda$. The following statements are equivalent.

(a) $\Lambda$ is of finite representation type.

(b) $Q$ is of Dynkin type, that is, its underlying graph belongs to the following list.

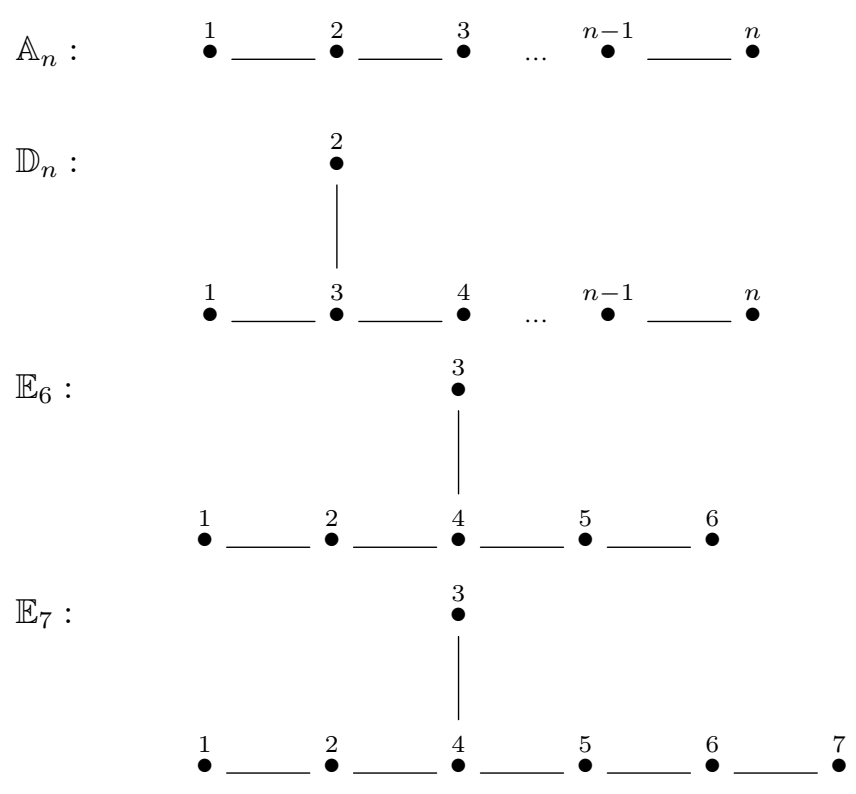




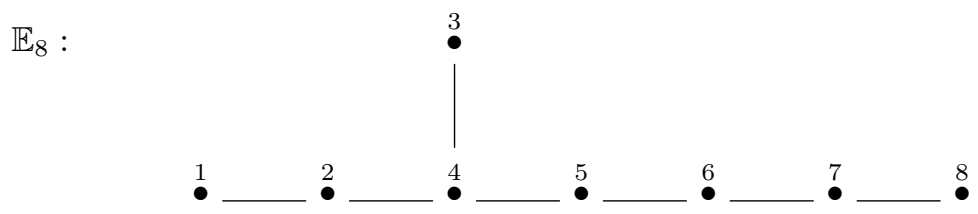

As we said in the introduction of this paper, concerning with preradicals over artin algebras of finite representation type, we can focus on their behavior only on the finite set $\Lambda$-ind of the indecomposable finitely generated modules. This leads us to study just the finite-dimensional representations of the quiver associated to the artin algebra.

The following concepts can be found in [3, V.5, V.7 and VII]. Let $M$ and $N$ be modules. A homomorphism $f: M \rightarrow N$ is an irreducible morphism if $f$ is neither a split monomorphism nor a split epimorphism, and if $f=h k$ for some $h: M \rightarrow X$ and $k: X \rightarrow N$ then $h$ is a split monomorphism or $k$ is a split epimorphism. Let $\Lambda$ be an artin algebra and let $M, N \in \Lambda$-mod. The radical of $\operatorname{Hom}_{\Lambda}(M, N)$ is defined $\operatorname{as}_{\operatorname{rad}_{\Lambda}}(M, N)=\left\{f \in \operatorname{Hom}_{\Lambda}(M, N) \mid h f g\right.$ is not an isomorphism for any $X \in \Lambda$-ind, $g: X \rightarrow M, h: N \rightarrow X\}$.

We recall the following result.

Proposition 4.4. Let $k$ be an algebraically closed field. Let $Q$ be a quiver of Dynkin type, $\Lambda=k Q$ and $M, N \in \Lambda$-ind. Then:

(1) $\operatorname{End}_{\Lambda}(M) \cong k$.

(2) Every submodule of $M$ is fully invariant.

(3) Each $f \in \operatorname{Hom}_{\Lambda}(M, N)$ is a sum of compositions of irreducible morphisms.

Example 4.5. Let $\mathbb{A}_{n}$ be the oriented quiver:

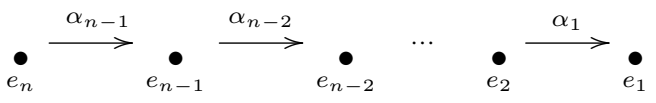

Let $k$ be a field and $\Lambda=k \mathbb{A}_{n} \cong\left(\begin{array}{ccc}k & \cdots & 0 \\ \vdots & \ddots & \vdots \\ k & \cdots & k\end{array}\right)$. A complete set of representatives of the isomorphism classes of indecomposable projective $\Lambda$-modules is $\left\{P_{1}, P_{2}, \ldots, P_{n}\right\}$ where:

$P_{1}=\Lambda e_{1}=k e_{1}$,

$P_{2}=\Lambda e_{2}=k e_{2}+k \alpha_{1}$,

$P_{3}=\Lambda e_{3}=k e_{3}+k \alpha_{2}+k \alpha_{1} \alpha_{2}$, 


$$
P_{n}=\Lambda e_{n}=k e_{n}+k \alpha_{n-1}+k \alpha_{n-2} \alpha_{n-1}+\cdots+k \alpha_{1} \alpha_{2} \cdots \alpha_{n-1} .
$$

The AR-quiver of $\Lambda$ is:

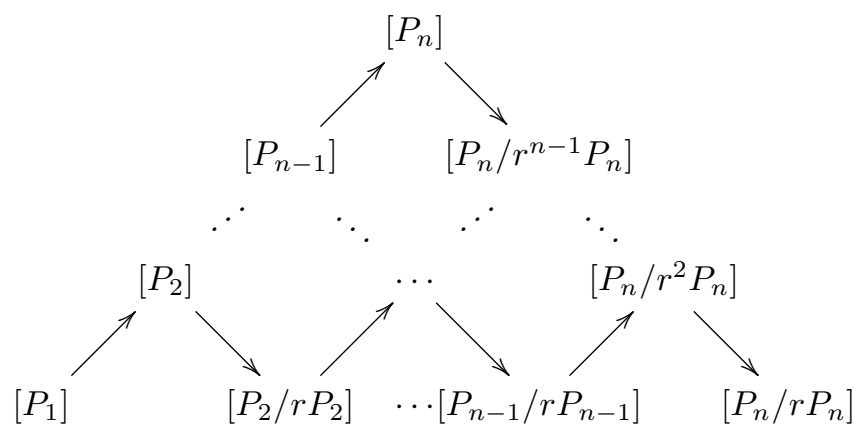

For each $\Lambda$-module $P_{i}$ we denote by $r P_{i}$ the Jacobson radical of $P_{i}$. Observe that for each $1 \leq i \leq n$ the composition length of $P_{i}$ is $c\left(P_{i}\right)=i$ and the lattice of submodules of $P_{i}$ is the chain $0=r^{i} P_{i}<r^{i-1} P_{i}<\cdots<r P_{i}<P_{i}$. The corresponding irreducible homomorphisms between representatives that appear in the AR-quiver are natural monomorphisms, corresponding to arrows that go upwards, and natural epimorphisms, corresponding to arrows that go downwards.

In view of Corollary 3.11, in the case of an artin algebra of finite representation type $\Lambda, \mathcal{I}$-compatible sets of submodules, and so, $\Lambda$-preradicals, can be determined by the AR-quiver $\Gamma_{\Lambda}$, as follows.

Corollary 4.6. Let $\Lambda$ be an indecomposable basic finite-dimensional hereditary algebra over an algebraically closed field $k$ of finite representation type. Let $N \in$ $\prod_{X \in \Lambda \text {-ind }} \mathcal{L}(X)$. Then the following conditions are equivalent.

(a) $N \in \mathcal{C}_{\Lambda-i n d}^{\Lambda}$.

(b) For each $X, Y \in \Lambda$-ind and each irreducible homomorphism $f: X \rightarrow Y$, $f\left(N_{X}\right) \leq N_{Y}$.

Proof. $(a) \Rightarrow(b)$ It is immediate.

$(b) \Rightarrow(a)$ Let $X, Y \in \Lambda$-ind and let $f: X \rightarrow Y$ be any homomorphism. If $X=Y$ then, since $\operatorname{End}_{\Lambda}(X) \cong k, f\left(N_{X}\right) \leq N_{X}$. On the other hand, if $X \neq Y$ then, by Proposition 4.4, $f$ is a sum of compositions of irreducible morphisms between indecomposable modules, so the hypothesis implies that $f\left(N_{X}\right) \leq N_{Y}$. We conclude that $N \in \mathcal{C}_{\Lambda-i n d}^{R}$.

Lemma 4.7. Let $R$ be a ring, and let $X$ be a left $R$-module of finite length. If $X$ has no repeated composition factors then $\mathcal{L}(X)$ is finite.

In particular, if $k$ is any field, and $\Lambda$ is the path $k$-algebra over a quiver of type $\mathbb{A}_{n}$ then $\mathcal{L}(X)$ is finite for any indecomposable $\Lambda$-module $X$. 
Proof. It follows easily by induction on the length of $X$.

Corollary 4.8. Let $k$ be an algebraically closed field and let $Q$ a quiver of type $\mathbb{A}_{n}$. Then the path algebra $k Q$ has a finite lattice of preradicals.

Proof. The number of appearances of the $n$ different simple modules as composition factors of a given indecomposable modules over $k Q$ is classified by the positive roots of the Tits integral quadratic form, in $n$ variables $x_{1}, \ldots, x_{n}, q\left(x_{1}, \ldots, x_{n}\right)=$ $\sum_{i=1}^{n} x_{i}^{2}-\sum_{i=1}^{n-1} x_{i} x_{i+1}$. It is well known that all such roots have only 0 and 1 as entries. By Lemma 4.7, each indecomposable module over $k Q$ has a finite lattice of submodules and, by Theorem 4.3, it has finite representation type. By Proposition 3.10 , the lattice of preradicals of $k Q$ is finite.

Lemma 4.9. Let $R$ be a left hereditary ring. Let $S$ be a simple left $R$-module such that its injective hull $E$ has finite length. Then $S$ appears only once as a composition factor of $E$.

Proof. Let $F$ be a submodule of $E$ such that $\operatorname{Soc}(E / F) \subseteq S$. As $R$ is left hereditary, $E / F$ is injective so it contains an injective hull of $S$ as a direct sum. As the injective hull of a module is unique up to isomorphism, we deduce that length $(E / F) \geq \operatorname{length}(E)$. Hence $F=0$, which implies that $S$ appears only once as a composition factor of $E$.

Proposition 4.10. Let $R$ be a left hereditary ring, and let $S$ be a simple left $R$ module such that its injective hull $E(S)$ has finite length. Fix $n \geq 0$. If $M$ is a left $R$-module of finite length such that $S$ appears $n$ times as a composition factor of $M$, then there exists $N \leq M$ such that $\operatorname{Soc}(M / N) \supseteq S^{n}$.

Proof. If $n=0$ then take $N=0$. Assume that $n>0$ and that the statement is true for modules of finite length such that $S$ appears $m$ times as a composition factor where $m<n$.

Let $K$ be a submodule of $M$ minimal with respect to the property that $\operatorname{Soc}(M / K)$ contains a submodule isomorphic to $S$. The minimality of $K$ implies that $M / K$ has $n$ composition factors isomorphic to $S$. Hence, we can assume that $S \subseteq \operatorname{Soc}(M)$, and we can fix a decomposition $E(M)=E(S) \oplus E$, where $E(M)$ denotes the injective hull of $M$.

Let $\pi: M \rightarrow E(S)$ denote the restriction of the canonical projection. By Lemma 4.9, $\pi(M)$ only has one composition factor isomorphic to $S$, therefore Ker $\pi$ has $n-1$ composition factors isomorphic to $S$. By the inductive hypothesis, there exists $N \leq \operatorname{Ker} \pi$ such that $\operatorname{Soc}(\operatorname{Ker} \pi / N)$ contains a submodule $X$ isomorphic to $S^{n-1}$. Then $\operatorname{Soc}(M / N)$ contains $X \oplus S$ which is isomorphic to $S^{n}$. 
Corollary 4.11. Let $k$ be an infinite field. Let $\Lambda$ be a hereditary finite dimensional $k$-algebra such that it has an indecomposable module of finite length $M$ such that

(i) $\operatorname{End}_{\Lambda}(M) \cong k$, that is, $M$ is a brick;

(ii) a composition series of $M$ has repeated factors.

Then the lattice of preradicals of Mod- $\Lambda$ is infinite.

Proof. Condition (i) ensures that all submodules of $M$ are invariant. Since $\Lambda$ is finite dimensional, the injective hull of any simple left $\Lambda$-module has finite length so that we can apply Proposition 4.10 to deduce that there exists $N \leq M$ such that $\operatorname{Soc}(M / N) \supseteq S^{n}$ for some simple module $S$ and some $n \geq 2$. Since $k$ is infinite, the lattice of submodules of $M$ containing $N$ is also infinite. Now for each one of these submodules we can construct the preradicals in Definition 2.3, this yields an infinite family of preradicals in Mod- $\Lambda$.

Proposition 4.12. Let $k$ be a field, and let $Q$ be a quiver of Dynkin type. The algebra $\Lambda=k Q$ has no indecomposable modules with repeated composition factors in its composition series if and only if $Q$ is of type $\mathbb{A}_{n}$.

Proof. If $Q$ is of type $\mathbb{A}_{n}$ then, as discussed in the proof of Corollary 4.8, the indecomposable $\Lambda$-modules satisfy the desired property.

Now suppose that $Q$ is not of type $\mathbb{A}_{n}$. We have the following cases, assuming that the vertices of $Q$ are numbered as in Theorem 4.3. If $Q$ is of type $\mathbb{D}_{n}$ then the Tits quadratic form of $Q$ is $q_{Q}(x)=\sum_{i=1}^{n} x_{i}^{2}-x_{1} x_{3}-\sum_{i=2}^{n-1} x_{i} x_{i+1}$ and $x=$ $(1,1,2,1, \ldots, 1)$ is a positive root of $q_{Q}$. If $Q$ is of type $\mathbb{E}_{6}$ then $x=(1,1,1,2,1,1)$ is a positive root of $q_{Q}(x)=\sum_{i=1}^{6} x_{i}^{2}-x_{1} x_{2}-x_{2} x_{4}-x_{3} x_{4}-x_{4} x_{5}-x_{5} x_{6}$. Similarly, if $Q$ is of type $\mathbb{E}_{7}$ then $x=(1,1,1,2,1,1,1)$ is a positive root of $q_{Q}(x)=\sum_{i=1}^{7} x_{i}^{2}-$ $x_{1} x_{2}-x_{2} x_{4}-\sum_{i=3}^{6} x_{i} x_{i+1}$, and if $Q$ is of type $\mathbb{E}_{8}$ then $x=(1,1,1,2,1,1,1,1)$ is a positive root of $q_{Q}(x)=\sum_{i=1}^{8} x_{i}^{2}-x_{1} x_{2}-x_{2} x_{4}-\sum_{i=3}^{7} x_{i} x_{i+1}$. Therefore in all cases there is a finite length module over $\Lambda$ with repeated composition factors.

Now we are ready to prove a characterization of hereditary finite-dimensional algebras with a finite lattice of preradicals.

Theorem 4.13. Let $\Lambda$ be a finite-dimensional hereditary algebra over an algebraically closed field $k$. Then $\Lambda$-pr is finite if and only if its basic subalgebra is a product of indecomposable algebras each one having Gabriel quiver of type $\mathbb{A}_{n}$.

Proof. Assume that $\Lambda$-pr is finite. Since $\Lambda$ is Morita equivalent to its basic subalgebra, me may assume that $\Lambda$ is basic. A basic artin algebra is a finite product of indecomposable basic artin algebras, thus we may also assume that $\Lambda$ is basic, 
indecomposable and hereditary (see Examples 2.6 (1) and (2)). Then, by Gabriel's Theorem $4.2, \Lambda \cong k Q$ for a suitable connected, acyclic quiver $Q$.

First we claim that $Q$ is a tree. If this is not the case then, by [1, proof of Proposition VII.2.3], $\lambda$ has a family $\left\{M_{a}\right\}_{a \in k}$ of finite dimensional $\Lambda$-modules with endomorphism ring isomorphic to $k$ and $\operatorname{Hom}_{\Lambda}\left(M_{a}, M_{b}\right)=\{0\}$ for $a \neq b$. Therefore the preradicals $\alpha_{M_{a}}^{M_{a}}$ (see Definition 2.3) give an infinite family of preradicals in $\Lambda$-pr, which is a contradiction. Hence $Q$ is a tree.

If $Q^{\prime}$ is a subquiver of $Q$ then there is a full and faithful embedding of $k Q^{\prime}$ - Mod into $\Lambda$ - Mod [1, Lemma VII.2.2]. Hence any preradical $\sigma$ over $k Q^{\prime}$ can be extended to a preradical over $\Lambda$, namely $\bigvee_{M \in k Q^{\prime} \text {-Mod }} \alpha_{\sigma M}^{M}$. In view of Proposition 4.12 and Corollary 4.11, this implies that $Q$ cannot contain a subquiver of type $\mathbb{D}_{n}$ for $n \geq 4$. Hence, $Q$ is of type $\mathbb{A}_{n}$.

\section{The lattice of preradicals of a specific path algebra}

In this section we study the finite lattice of preradicals over $\Lambda=k Q$, where $k$ is an algebraically closed field and $Q$ is the quiver of Dynkin type $\mathbb{A}_{n}$ with the canonical orientation, as in example 4.5. First we recall that $\Lambda$ is a left pure semisimple ring, where (see Theorem 3.2) $\mathcal{I}=\Lambda$-ind can be taken as the set $\left\{X_{i, j} \mid 1 \leq i \leq j \leq n\right\}$, where $X_{i, j}=P_{n+i-j} / r^{i} P_{n+i-j}$, as in the AR-quiver of Example 4.5. We have the following properties of these $\Lambda$-modules, including those stated in Corollary 4.4.

Recall that a module is called uniserial if its lattice of submodules is a chain. If $X$ is a module of finite length, the denote by $c(X)$ its composition length.

Proposition 5.1. For each $1 \leq i \leq j \leq n$ let $X_{i, j}$ as above. Then:

(1) $X_{i, j}$ is a uniserial module.

(2) $\operatorname{End}\left(X_{i, j}\right) \cong k$.

(3) Every submodule of $X_{i, j}$ is fully invariant.

(4) $c\left(X_{i, j}\right)=i$.

We consider irreducible homomorphisms between elements of $\mathcal{I}$ as follows.

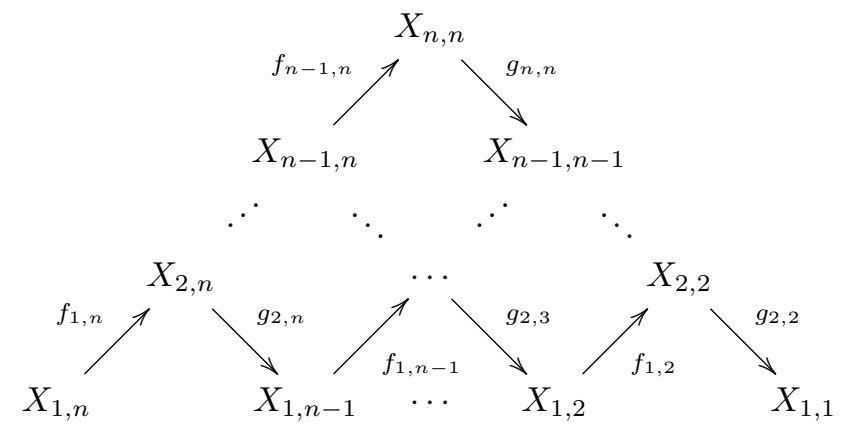


For each $i, j$ the $f_{i, j}$ are natural inclusions and the $g_{i, j}$ are natural epimorphisms.

Let $T_{n}$ be the set of all triangular arrays

$$
\left[c_{i, j}\right]=\left[\begin{array}{ccccc}
c_{n, n} & c_{n-1, n-1} & \cdots & c_{2,2} & c_{1,1} \\
c_{n-1, n} & c_{n-2, n-1} & \cdots & c_{1,2} & \\
\vdots & \vdots & . & & \\
c_{2, n} & c_{1, n-1} & & & \\
c_{1, n} & & & &
\end{array}\right]
$$

with entries $c_{i, j} \in \mathbb{N}$ for $1 \leq i \leq j \leq n$ satisfying the following conditions:

(1) $0 \leq c_{i, j} \leq i$

(2) $i<j \Rightarrow c_{i, j} \leq c_{i+1, j}$

(3) $i, j \geq 2 \Rightarrow c_{i, j} \leq c_{i-1, j-1}+1$.

In $T_{n}$ we can define order, join and meet coordinate-wise. In other words:

$\left[c_{i, j}\right] \leq\left[c_{i, j}^{\prime}\right] \Leftrightarrow c_{i, j} \leq c_{i, j}^{\prime}$, for every $1 \leq i \leq j \leq n ;$

$\left[c_{i, j}\right] \vee\left[c_{i, j}^{\prime}\right]=\left[\max \left\{c_{i, j}, c_{i, j}^{\prime}\right\}\right]$;

$\left[c_{i, j}\right] \wedge\left[c_{i, j}^{\prime}\right]=\left[\min \left\{c_{i, j}, c_{i, j}^{\prime}\right\}\right]$.

This defines a lattice $\left\langle T_{n}, \leq, \vee, \wedge\right\rangle$ with least element $\left[z_{i, j}\right]$ and greatest element $\left[u_{i, j}\right]$, where for each $1 \leq i \leq j \leq n, z_{i, j}=0$ and $u_{i, j}=i$.

Proposition 5.2. The lattices $\Lambda$-pr and $T_{n}$ are isomorphic.

Proof. By Proposition 3.10 it is enough to prove that the lattices $\mathcal{C}_{\mathcal{I}}^{\Lambda}$ and $T_{n}$ are isomorphic. For simplicity, for each $Y \in \prod_{1 \leq i \leq j \leq n} \mathcal{L}\left(X_{i, j}\right)$ we write $Y_{i, j}$ instead of $Y_{X_{i, j}}$. Let us consider the function $\varphi: \mathcal{C}_{\mathcal{I}}^{\Lambda} \rightarrow T_{n}$ such that $\varphi(Y):=\left[c_{i, j}\right]$, where for each $1 \leq i \leq j \leq n, c_{i, j}=c\left(Y_{i, j}\right)$. Therefore we have for each $1 \leq i \leq$ $j \leq n$ that $0 \leq c_{i, j} \leq i$, which is condition (1) stated above. Now, if $i<j$ then $f_{i, j}\left(Y_{i, j}\right) \leq Y_{i+1, j}$. Since $f_{i, j}$ is a monomorphism then $c\left(f_{i, j}\left(Y_{i, j}\right)\right)=c_{i, j}$. Therefore $c_{i, j} \leq c_{i+1, j}$, which is condition (2). Finally, if $i, j \geq 2$ then $g_{i, j}\left(Y_{i, j}\right) \leq Y_{i-1, j-1}$. By definition of $g_{i . j}$ we have $c\left(g_{i, j}\left(Y_{i, j}\right)\right)=c_{i, j}-1$. Therefore $c_{i, j}-1 \leq c_{i-1, j-1}$, which is condition (3). This proves that $\left[c_{i, j}\right] \in T_{n}$.

Now we define the function $\psi: T_{n} \rightarrow \mathcal{C}_{\mathcal{I}}^{\Lambda}$ as follows. For each $\left[c_{i, j}\right] \in T_{n}$ there is a unique set $Y \in \prod_{1 \leq i \leq j \leq n} \mathcal{L}\left(X_{i, j}\right)$ such that $c\left(Y_{X_{i, j}}\right)=c_{i, j}$, for each $1 \leq i \leq j \leq n$. We claim that $Y \in \mathcal{C}_{\mathcal{I}}^{\Lambda}$. To prove this we use Corollary 4.6. So let $h: X \rightarrow X^{\prime}$ be an irreducible morphism, where $X, X^{\prime} \in \Lambda$-ind. Then there must be an arrow $[X] \rightarrow\left[X^{\prime}\right]$ in the AR-quiver of $\Lambda$ so that either $X=X_{i, j}$ and $X^{\prime}=X_{i+1, j}$ for some $1 \leq i<j \leq n$, or $X=X_{i, j}$ and $X^{\prime}=X_{i-1, j-1}$ for some $2 \leq i \leq$ $j \leq n$. In the first case, $h$ must be a monomorphism, so that $h\left(Y_{i, j}\right) \cong Y_{i, j}$ has 
composition length $c_{i, j} \leq c_{i+1, j}$, due to the fact that $\left[c_{i, j}\right] \in T_{n}$. Since every $X_{i, j}$ is uniserial we must have $h\left(Y_{i, j}\right) \leq Y_{i+1, j}$. In the second case $h$ must be an epimorphism, so that $X_{i, j} / \operatorname{Ker}(f) \cong X_{i-1, j-1}$. Furthermore, $c(\operatorname{Ker}(f))=1$, because $c\left(X_{i-1, j-1}\right)=i-1=c\left(X_{i, j}\right)-1$. If $h\left(Y_{i, j}\right)=0$, we are done, so we can assume that $\operatorname{Ker}(f)<Y_{i, j}$, since $X_{i, j}$ is uniserial. Therefore $h\left(Y_{i, j}\right) \cong Y_{i, j} / \operatorname{Ker}(f)$, so $c\left(h\left(Y_{i, j}\right)\right)=c_{i, j}-1 \leq c_{i-1, j-1}$, since $\left[c_{i, j}\right] \in T_{n}$. This proves that in this case $h\left(Y_{i, j}\right) \leq Y_{i-1, j-1}$, so in any way $h\left(Y_{X}\right) \leq Y_{X^{\prime}}$ and we conclude that $Y \in \mathcal{C}_{\mathcal{I}}^{\Lambda}$.

It is obvious that $\varphi$ and $\psi$ are order (and hence lattice) isomorphisms inverse one to each other.

Theorem 5.3. Let $\Lambda=k Q$, where $k$ is an algebraically closed field and $Q$ is the quiver of Dynkin type $\mathbb{A}_{n}$ with the canonical orientation. Then:

Claim 1. $\Lambda$-pr is a finite distributive lattice.

Proof. That $\Lambda$-pr is finite is a consequence of Corollary 4.6. On the other hand, by Proposition 5.2, $\Lambda$-pr $\cong \mathcal{C}_{\mathcal{I}}^{\Lambda}$, a sublattice of the lattice $\prod_{1 \leq i \leq j \leq n} \mathcal{L}\left(X_{i, j}\right)$, which is distributive, being a product of chains. Therefore $\Lambda$-pr is distributive.

Claim 2. $\Lambda$-pr is a self-dual lattice.

Proof. By Proposition 5.2 it is enough to prove that $T_{n}$ is a self-dual lattice. Let $\left[c_{i, j}\right] \in T_{n}$. For each $1 \leq i \leq j \leq n$ let $c_{i, j}^{*}=i-c_{i, n+i-j}$. We claim that $\left[c_{i, j}^{*}\right] \in T_{n}$. If $1 \leq i \leq j \leq n$ then $0 \leq c_{i, n+i-j} \leq i$, hence we have $0 \leq c_{i, j}^{*} \leq i$, which is condition (1). If $i<j$ then, since $\left[c_{i, j}\right]$ satisfies condition (3) we have $c_{i+1, n+i+1-j} \leq$ $c_{i, n+i-j}+1$. Therefore $c_{i, j}^{*}=i-c_{i, n+i-j} \leq i+1-c_{i+1, n+i+1-j}=c_{i+1, j}^{*}$, so that $\left[c_{i, j}^{*}\right]$ satisfies condition (2). Now suppose that $i, j \geq 2$. Since $\left[c_{i, j}\right]$ satisfies condition (2) we have $c_{i-1, n+i-j} \leq c_{i, n+i-j}$. Therefore $c_{i, j}^{*}=i-c_{i, n+i-j} \leq i-c_{i-1, n+i-j}=$ $c_{i-1, j-1}^{*}+1$, and $\left[c_{i, j}^{*}\right]$ satisfies condition (3). We conclude that $\left[c_{i, j}^{*}\right] \in T_{n}$. Therefore we can define the function $\delta: T_{n} \rightarrow T_{n}$ such that $\delta\left(\left[c_{i, j}\right]\right):=\left[c_{i, j}^{*}\right]$. Notice that for each $1 \leq i \leq j \leq n$ we have $c_{i, j}^{* *}=c_{i, j}$, so that $\delta^{2}=1_{T_{n}}$. Notice also that $\delta$ reverses order. We conclude that it is an order anti-isomorphism.

Claim 3. $\Lambda$-pr is a graded lattice with rank $\sum_{i=1}^{n} \frac{i(i+1)}{2}$.

Proof. By Proposition 5.2 it is enough to prove that $T_{n}$ is a graded lattice. Let $\rho: T_{n} \rightarrow \mathbb{N}$ be such that $\rho\left(\left[c_{i, j}\right]\right):=\sum_{1 \leq i \leq j \leq n} c_{i, j}$. Then $\rho\left(\left[z_{i, j}\right]\right)=0$. Suppose that $\mathbf{c}=\left[c_{i, j}\right], \mathbf{d}=\left[d_{i, j}\right] \in T_{n}$ are such that $\mathbf{d}$ covers $\mathbf{c}$. Then there exist $1 \leq k \leq l \leq n$ such that $d_{k, l}=c_{k, l}+1$ and for each $1 \leq i \leq j \leq n$ with $k \neq i$ and $l \neq j$ we have $d_{i, j}=c_{i, j}$. Therefore $\rho\left(\left[d_{i, j}\right]\right)=\rho\left(\left[c_{i, j}\right]\right)+1$. We conclude, by Theorem 2.1, that $T_{n}$ is a graded lattice with $\operatorname{rank} \rho\left(\left[u_{i j}\right]\right)=\sum_{1 \leq i \leq j \leq n} u_{i, j}=\sum_{i=1}^{n} \frac{i(i+1)}{2}$. 


\section{References}

[1] I. Assem, D. Simson and A. Skowronski, Elements of the Representation Theory of Associative Algebras, Vol.1, London Mathematical Society Student Texts, 65, Cambridge University Press, Cambridge, 2006.

[2] M. Auslander, Representation theory of Artin algebras II, Comm. Algebra, 1 (1974), 269-310.

[3] M. Auslander, I. Reiten and S. O. Smalø, Representation Theory of Artin Algebras, Cambridge Studies in Advanced Mathematics, 36, Cambridge University Press, Cambridge, 1997.

[4] L. Bican, T. Kepka and P. Nemec, Rings, Modules and Preradicals, Lecture Notes in Pure and Applied Mathematics, 75, Marcel Dekker, Inc., New York, 1982.

[5] G. Birkhoff, Lattice Theory, Colloquium Publications XXV, American Mathematical Society, New York, 1948.

[6] D. Eisenbud and P. Griffith, The structure of serial rings, Pacific J. Math., 36 (1971), 109-121.

[7] R. Fernández-Alonso and S. Gavito, The lattice of preradicals over local uniserial rings, J. Algebra Appl., 5(6) (2006), 731-746.

[8] P. Gabriel, Unzerlegbare Darstellungen I, Manuscripta Math., 6 (1972), 71-103.

[9] G. Grätzer, General Lattice Theory, Birkhäuser Verlag, Basel, 2003.

[10] L. Gruson and C. U. Jensen, Deux applications de la notion de L-dimension, C. R. Acad. Sci. Paris, Sér. A-B, 282(1) (1976), 23-24.

[11] B. Huisgen-Zimmermann, Purity, algebraic compactness, direct sum decompositions and representation type, Infinite length modules, Trends Math., (2000), 331-367.

[12] F. Raggi, J. R. Montes, H. Rincón, R. Fernández-Alonso and C. Signoret, The lattice structure of preradicals, Comm. Algebra, 30(3) (2002), 1533-1544.

[13] F. Raggi, J. Ríos, H. Rincón, R. Fernández-Alonso and C. Signoret, The lattice structure of preradicals II: Partitions, J. Algebra Appl., 1(2) (2002), 201-214.

[14] R. P. Stanley, Enumerative Combinatorics, Vol. I, Wadsworth and Brooks/Cole Advanced Books and Software, Monterey, CA, 1986.

[15] B. Stenström, Rings of Quotients, Die Grundlehrem der Mathematischen Wissenschaften 217, Springer-Verlag, New York-Heidelberg, 1975.

[16] B. Zimmermann-Huisgen and W. Zimmermann, On the sparsity of representations of rings of pure global dimension zero, Trans. Amer. Math. Soc., 320(2) (1990), 695-711. 
Rogelio Fernández-Alonso (Corresponding Author)

Departamento de Matemáticas

Universidad Autónoma Metropolitana - Iztapalapa

San Rafael Atlixco 186, Ciudad de México

e-mail: rfg@xanum.uam.mx

\section{Dolors Herbera}

Departament de Mathematiques

Universitat Autònoma de Barcelona

08193 Bellaterra, Barcelona, Spain

e-mail: dolors@mat.uab.cat 